\title{
Inspiratory muscle training improves strength and health-related quality of life in hemodialysis patients
}

\author{
Viviane Soares ${ }^{(1)}$, Iransé Oliveira-Silva(1), Maria Sebastiana Silva ${ }^{(2)}$, Patrícia Espíndola Mota Venâncio(1), \\ Luís Vicente Franco Oliveira ${ }^{(1)}$, William Alves Lima', Jairo Teixeira Júnior ${ }^{(1)}$, Grassyara Pinho Tolentino(1), \\ Thailyne Bizinotto ${ }^{(1)}$.
}

\begin{abstract}
Background: Patients undergone hemodialysis (HD) suffer with energy-protein malnutrition, uremic myopathy and protein catabolism reducing their functional capacity, tolerance to exercise and aspects related to quality of life. Objective: The aim of this study was to evaluate the effects of the two protocols of inspiratory muscle training (IMT) on muscle strength, pulmonary function and related-health quality of life on male HD patients. Methods: Maximal inspiratory pressure (MIP) and maximal expiratory pressure (MEP), peak expiratory flow (PEF), forced expiratory volume in first second $\left(F_{1} V_{1}\right)$, forced vital capacity $(F V C)$ and the $F E V_{1} / F V C$ ratio and quality of life were evaluated pre and post six months of IMT in HD patients. IMT was performed by one group using a specific training (Power breathe) device (T-IMT, $n=19)$ and by the other with incentive spirometry (I-IMT, $n=15)$. Results: Following the inspiratory training, muscle strength improved in both groups. The group that used incentive spirometry had increments in MIP larger (39.8\%, $<<0.001)$ than the group that trained with the specific training device $(28.3 \%, p=0.001)$. The KDQOL-SF dimensions showed significant difference in the T-IMT group in cognitive function $(p=0.03)$, sexual function $(p=0.009)$ and social function dimensions $(p=0.04)$ and in the I-IMT group in the physical function dimension $(\mathrm{p}=0.03)$. Conclusion: It was proved that IMT improved muscle strength and I-IMT promoted increments significantly larger in MIP. Aspects of health-related quality of life of HD male patients improved significantly with T-IMT (sexual, social and cognitive functions), while I-IMT ameliorated only physical function.
\end{abstract}

Key-words: Breathing Exercises, Exercise Therapy, Quality of Life, Hemodialysis.

\section{INTRODUCTION}

Several factors, including energy-protein malnutrition, increase in the parathyroid hormone (PTH), abnormalities in vitamin D activity, uremic myopathy and protein catabolism cause impairment in the physical and functional capacity of patients undergoing hemodialysis (HD) treatment ${ }^{(1-8)}$. Moreover, studies have shown that changes occur in the morphological and histochemical aspects of muscles, such as the reduced cross-sectional area of the fiber, atrophy and reduction in size of fiber type I and type II, infiltration and degeneration of muscle fiber, production of actin fragments of $14 \mathrm{kD}$ (kiloDalton) and reduction in the synthesis of mitochondrial proteins and inside the oxidative capacity of muscle cells, further decreasing muscle capillary density (7-9). As a consequence, respiratory muscle strength, pulmonary function and exercise capacity are affected. However, studies have also demonstrated that inspiratory muscle training (IMT) programs may benefit respiratory (increasing muscle strength) and heart function (increasing cardiac output and oxygen consumption), as well as an improvement in managing everyday activities and the functional capacity of these patients $s^{(7,8)}$.

It is known that patients on HD present deficits in muscle strength and pulmonary function ${ }^{(10-12)}$. Weiner et al. ${ }^{(10)}$ showed that inspiratory muscle performance significantly increased, and was associated with improvement in, functional capacity. Another study performed inspiratory muscle training for eight weeks in 15 patients and found no increase in inspiratory muscle pressure (MIP) ${ }^{(11)}$. In contrast, the results of a randomized controlled study showed that 10 weeks of IMT improved inspiratory strength and functional performance, but that pulmonary function did not benefit by this training ${ }^{(12)}$. Thus there is little conclusive evidence in the literature on IMT in HD patients and we hypothesized that six months of IMTcan improve respiratory variables and aspects related to quality of life of HD patients. The aim of this study was to evaluate the effects of the two protocols of IMT on muscle strength, pulmonary function and Health-related quality of life (HRQL) on male HD patients. 


\section{METHODS}

\section{Study design}

This study was a prospective clinical trial performed by simple randomization.

\section{Patients}

The study enrolled from January 2012 to August 2013. One hundred thirteen male patients in HD from two dialysis centers in Goiânia, Goiás, Brazil were evaluated for eligibility of study. Of these 64 held to intervention (figure 1). All patients were submitted to dialysis three times per week for 3-4 hours/day coupled to a dialysis machine (Baxter, USA) by means of an arteriovenous fistula. The dialyzer or capillary filter (Xenium, Japan) had high efficiency and was composed of cellulose diacetate membrane (it has a large number of free hydroxyl groups on the surface) with features of biocompatibility. The inclusion criteria were older than 18 years, treatment time exceeding three months, three times per week for 3-4 hours/day, and exclusion criteria were chronic obstructive pulmonary disease (COPD), uncontrolled cardiac arrhythmia, unstable angina, other severe cardiac disease, decompensated blood pressure (systolic blood pressure $>180 \mathrm{mmHg}$ and diastolic blood pressure $>100 \mathrm{mmHg}$ ), diabetes mellitus (serum glucose $>110 \mathrm{mg} / \mathrm{dL}$ ), subjects with clear inability to use a respiratory device or to collaborate. The study was approved by the Ethics Committee in Research of the Universidade Federal de Goiás (Brazil), under protocol number
$294 / 2011$. Those who participated in the study signed an informed consent.

They were allocated into three groups by simple randomization using sealed envelopes containing the patients' names. The nurse manager randomly selected them and the patients were alternately allocated to each group. One group underwent training using a specific trainer (T-IMT), other group performed two breathing exercises and incentive spirometry (I-IMT) during HD and control group. The respiratory muscle strength and pulmonary function tests were performed on no-dialysis days and at an intermediate session in the week. All tests were performed pre-, mid- (three months) and post-IMT that lasted six months in total. The KDQOL-SF questionnaire was applied pre and post.

\section{Protocols}

A record was filled out with information about age, time on $\mathrm{HD}$, body mass, height, medication used and smoking history. The hematocrit, hemoglobin, urea pre- and post-, phosphorus, potassium and the adequacy of dialysis parameters were collected from the patient's medical record in the first week of each month for six month in order to monitor anemia. The adequacy of dialysis (Kt/V) was calculated using the Daugirdas formula ${ }^{(13)}$.

Respiratory muscle strength was measured by MIP and MEP which were held from residual volume and total lung capacity against an occluded airway with a minor air leak $(2 \mathrm{~mm})$ respectively ${ }^{(15,16)}$. The maneuvers were explained and demonstrated to each patient. Patients remained seated with the airway occluded by a nose clip and a

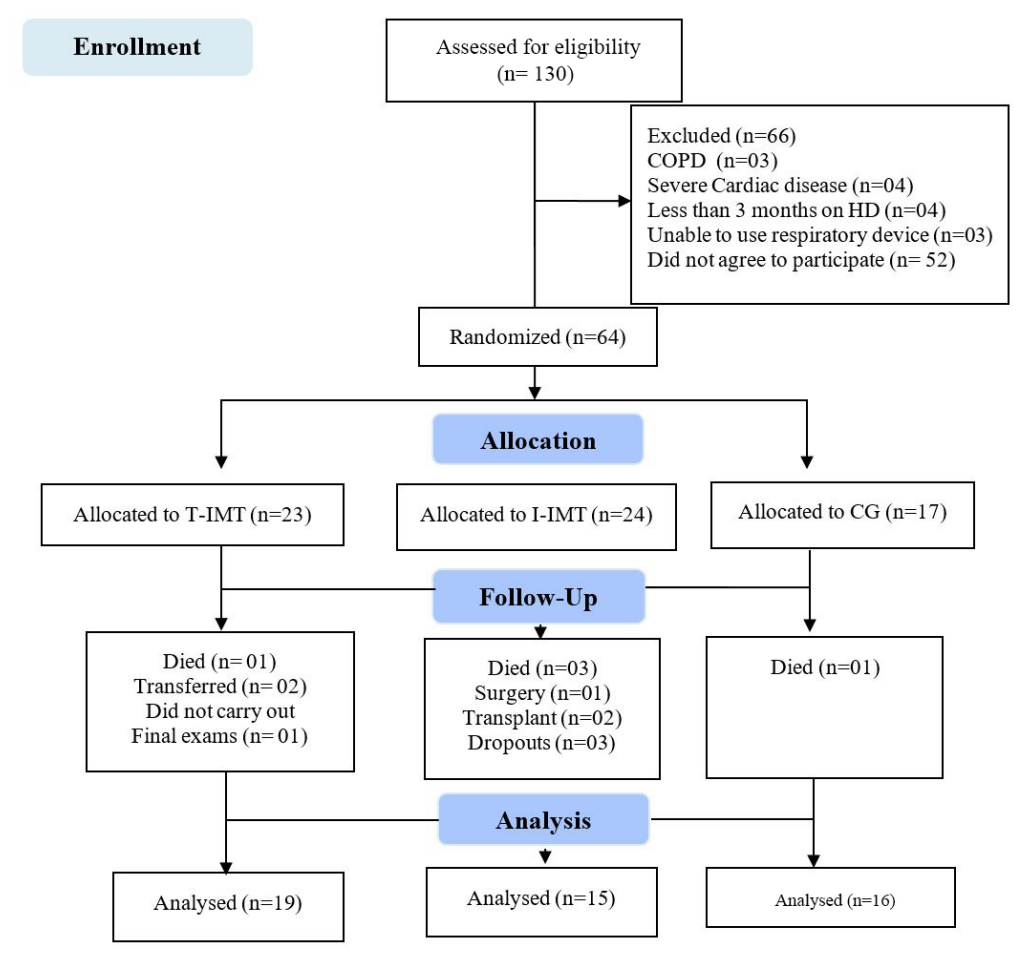

Figure 1- Study design 
mouthpiece connected to a manometer (Globalmed, Porto Alegre, Brazil). Inspiratory and expiratory efforts were sustained for one second (s). Three to five acceptable and reproducible maneuvers (difference less than or equal to $10 \%$ ) were performed and the biggest value was selected. The interval between maneuvers was one minute for all those tested. The predicted values were calculated according to Neder et al. ${ }^{(17)}$.

Spirometrics variables were collected with a computerized spirometer (Micro quark, Cosmed, Rome, Italy). To perform the test and interpretation we used the criteria of the American Thoracic Society ${ }^{(18)}$. The analyzed variables were peak expiratory flow (PEF), forced expiratory volume in first second $\left(F E V_{1}\right)$, forced vital capacity $(F V C)$ and the $\mathrm{FEV}_{1} / \mathrm{FVC}$ ratio.

To evaluate health-self quality of life was used the KDQOL-SF (Kidney Disease Quality of Life - Short Form) questionnaire. The KDQOL-SF was read aloud and the patients followed the printed questionnaire visually, item-by-item. The questionnaire includes SF-36 (Medical Outcomes Study 36) with eight domains about physical and mental health and specific items about kidney disease. This instrument was validated in Brazil by Duarte et al. ${ }^{(14)}$. The scores range from 0 to 100 and the lower scores correspond to worse health-related quality of life and higher values reflect better quality of life.

\section{Inspiratory Muscle Training}

The IMT was carried out for six months, three times per week during the first two hours of hemodialysis. Patients remained seated or on the reclining hemodialysis' chair at 45․ Before the beginning of each exercise session, the clinical signs such as blood pressure, heart rate, breathing pattern and oxygen saturation were checked.

The T-IMT group trained with one specific trainer (Power Breathe, Southam, UK), and the I-IMT group with the incentive spirometer (Respiron, NCS, São Paulo, Brazil). Patients began training using $30.0 \%$ of MIP and performing three sets of 10 maximal inspirations with 60 seconds of rest time between them. The adjustment of the load, number of inspirations and number of series was changed each 30 days The mean time to execute the training was 11 minutes. The incentive spirometer (I-IMT) training group performed two breathing exercises, diaphragmatic breathing ${ }^{(20)}$ (for breath control) and inspiration in times (to increase tidal volume) ${ }^{(20,21)}$. The control group underwent the same assessments as the others groups without any intervention.

\section{Data analysis}

Data are expressed as frequencies, mean, standard deviation (SD), percentage or graphs. The Shapiro-Wilk test was used to verify normality. The differences between the two groups (T-IMT and I-IMT) were analyzed by t-Student non-paired for continuous variables and Chi-square for categorical variables. The effects of interventions on continuous variables were compared by ANOVA for repeated measures with Bonferroni adjustments. The t-student assessed the difference between the dimensions of the KDQOL-SF questionnaire. The data were processed and analyzed using the Statistical Package Social Sciences (SPSS, Chicago, US). Significance was accepted when the probability was $<0.05$.

\section{RESULTS}

The baseline characteristics are shown in Table 1. Of 47 patients enrolled, 13 did not complete the study (Figure 1). However, 34 male patients were studied with an average age of 51.3 (13.2) years (ranging from 19 to 75 years). $36.8 \%$ and $5.3 \%$ of the IMT-T group and $33.3 \%$ and $26.7 \%$ of the IMT-I group were classified as pre-obese and obese I, respectively. The etiology of renal disease $(p=0.47)$ and a history of smoking $(p=0.07)$ were not significantly associated with the type of intervention. The main cause of chronic kidney disease was hypertensive nephrosclerosis in the T-IMT group (42.0\%) and the I-IMT group (40.0\%) which were related to the use of anti-inflammatory hormones and infections.

Table 1. Clinical baseline characteristics of patients randomized to T-IMT, I-IMT and CG.

\begin{tabular}{|c|c|c|c|}
\hline & $\begin{array}{l}\text { T -IMT } \\
(n=19)\end{array}$ & $\begin{array}{l}\text { I-IMT } \\
(n=15)\end{array}$ & $\begin{array}{c}\text { CG } \\
(n=16)\end{array}$ \\
\hline Age (years) & $54.7(10.4)$ & $49.5(13.2)$ & $49.3(16.3)$ \\
\hline Body mass (kg) & 71.3 (10.9) & $74.6(12.7)$ & $68.8(14.9)$ \\
\hline Dry weight (kg) & 70.3 (10.9) & 73.5 (12.9) & $67.2(14.2)$ \\
\hline Height (m) & $1.7(0.04)$ & $1.7(0.08)$ & $1.7(0.09)$ \\
\hline $\mathrm{BMI}\left(\mathrm{kg} / \mathrm{m}^{2}\right)$ & $24.1(3.1)$ & $25.8(4.4)$ & $24.3(4.2)$ \\
\hline Time on HD (months) & $44.16(47.3)$ & $57.3(42.1)$ & $55.1(57.7)$ \\
\hline Hemoglobin (mg/dl) & $11.2(1.1)$ & $11.5(1.2)$ & $10.7(1.5)$ \\
\hline Hematocrit (\%) & $33.2(6.8)$ & $35.6(3.1)$ & $32.9(4.6)$ \\
\hline Urea-pre (mg/dl) & $172.4(56.8)$ & $170.1(47.4)$ & $147.1(33.3)$ \\
\hline Urea-post (mg/dl) & $47.1(21.5)$ & $42.7(16.8)$ & $45.8(17.2)$ \\
\hline Potassium (mg/dl) & $5.7(1.0)$ & $5.4(1.0)$ & $5.3(0.6)$ \\
\hline Phosphorus (mg/dl) & $5.4(1.5)$ & $5.6(1.8)$ & $5.8(1.6)$ \\
\hline $\mathrm{Kt} / \mathrm{V}$ & $1.7(0.4)$ & $1.8(0.3)$ & $1.5(0.4)$ \\
\hline \multicolumn{4}{|l|}{ Etiology of CKD - n (\%) } \\
\hline SAH & $08(42.0)$ & $05(33.4)$ & $07(43.8)$ \\
\hline DM & $04(21.1)$ & $02(13.3)$ & $01(6.3)$ \\
\hline CGN & $03(15.8)$ & $02(13.3)$ & $06(37.5)$ \\
\hline Others & $04(21.1)$ & $06(40.0)$ & $02(12.5)$ \\
\hline \multicolumn{4}{|l|}{ Smoking - n (\%) } \\
\hline Former smokers & 15 (78.9) & $06(40.0)$ & $11(68.8)$ \\
\hline Never smoked & $04(21.1)$ & $09(60.0)$ & $05(31.3)$ \\
\hline
\end{tabular}


Table 2- Variables of respiratory muscle strength and pulmonary function for the two groups pre-, mid- and post-IMT.

\begin{tabular}{|c|c|c|c|c|c|c|c|c|c|}
\hline \multirow{2}{*}{ Variables } & \multicolumn{3}{|c|}{ T-IMT (n=19) } & \multicolumn{3}{|c|}{ I-IMT (n=15) } & \multicolumn{3}{|c|}{ CG $(n=16)$} \\
\hline & Pre & Mid & Post & Pre & Mid & Post & Pre & Mid & Post \\
\hline MIP (cmH2O) & $67.2(27.7)$ & $79.4(24.4)^{*}$ & $86.2(26.8)^{+}$ & 71.7 (27.8) & $94.0(28.0)^{*}$ & $100.3(25.9)^{\dagger}$ & $77.2(26.0)$ & $84.8(28.5)$ & $88.4(26.3)$ \\
\hline $\operatorname{MEP}(\mathrm{cmH} 2 \mathrm{O})$ & $83.4(29.3)$ & $90.1(25.1)$ & $93.0(26.6)$ & $97.5(34.1)$ & $92.5(28.4)$ & $95.5(25.4)$ & $85.6(38.6)$ & $85.8(34.3)$ & $75.8(18.4)$ \\
\hline PEF (L/s) & $6.0(2.3)$ & $6.5(2.6)$ & $7.0(2.1)$ & $6.1(2.4)$ & $6.5(2.2)$ & $6.8(2.0)$ & $5.6(2.1)$ & $6.3(2.3)$ & $6.6(2.2)$ \\
\hline FEV1 (L) & $2.8(0.6)$ & $2.8(0.6)$ & $2.9(0.7)$ & $3.0(0.8)$ & $2.9(0.7)$ & $2.9(0.7)$ & $2.9(0.7)$ & $3.0(0.7)$ & $3.0(0.7)$ \\
\hline FVC (L) & $3.5(0.6)$ & $3.4(0.7)$ & $3.6(0.7)$ & $3.6(0.8)$ & $3.6(0.7)$ & $3.6(0.8)$ & $3.7(0.6)$ & $3.8(0.8)$ & $3.7(0.8)$ \\
\hline $\mathrm{FEV}_{1} / \mathrm{FVC}(\%)$ & $80.0(0.1)$ & $81.2(0.1)$ & $80.1(0.1)$ & $81.7(0.1)$ & $81.7(0.1)$ & $82.0(0.1)$ & $78.0(0.1)$ & $81.2(0.1)$ & $81.0(0.1)$ \\
\hline
\end{tabular}

Note: Data presented as mean \pm SD. T-IMT = trainer - inspiratory muscle training; I-IMT = incentive - inspiratory muscle training; MIP = maximal inspiratory pressure; MEP = maximal expiratory pressure; PEF = peak expiratory flow; FEV1 = forced expiratory volume in first second; FCV = forced capacity vital. "Difference between pre-mid; ${ }^{+}$difference between pre-post. Values of $p$ obtained to $p<0.05$.

The variables related to respiratory muscle strength and pulmonary function pre-, mid-, and post-intervention protocols are shown in Table 2. The MIP improved during and after in both T-IMT and I-IMT groups. The MIP values in the T-IMT group increased by $18.1 \%(p=0.008)$ and $28.3 \%(p=0.001)$ following three and six months of training respectively. In the I-IMT group, the increments were higher, attaining $31.0 \%(p=0.01)$ and $39.8 \%(p<0.001)$ after three and six months of inspiratory training respectively. Two patients in the T-IMT group and one in the I-IMT group had MIP predicted before the intervention. At the end of inspiratory training nine patients in T-IMT and seven in I-IMT had MIP values within the expected range for age and sex. I-IMT induced a significantly larger increase in MIP ( $p=0.02$ ) when compared to T-IMT. In contrast, no significant differences were found to MEP in either group. In addition, peak expiratory flow was the only variable of pulmonary function that showed differences pre- and post-training for T-IMT $(p=0.02)$.

The aspects related to HRQL assessed by KDQOL-SF showed significant differences in social function $(p=0.004)$, sexual function ( $p=0.009)$ and cognitive function $(p=0.03)$ dimensions in the T-IMT group, and in physical role $(p=0.03)$ in the I-IMT group (Table 3). The general health dimension was significantly reduced in the I-TMT group $(p=0.04)$. And, before the intervention the mean score of the burden of kidney disease and work dimensions remained below 50 points.

\section{DISCUSSION}

In this study, we showed that HD patients had reduced respiratory muscle strength and lung function when compared with the predicted values (Table 1). Following IMT, inspiratory muscle strength improved in both groups. Moreover, the group that used incentive spirometry showed a greater increase in strength than the T-IMT group. The scores of the aspects related to quality of life presented significant differences in the T-IMT group in cognitive function, sexual function and social function dimensions. In the I-IMT group, the physical function dimension and general health were the only that showed a significant difference after the intervention.

The involvement of the respiratory system in the clinical manifestations of CKD and its treatment is very complex but exercise training can help ameliorate the respiratory conditions of these patients. When evaluated before IMT the MIP and MEP values of patients in the T-IMT group were $5.3 \%$ and $15.8 \%$, respectively, of the values predicted for age and in the I-IMT group, the values were $6.7 \%$ and $13.3 \%$ for MIP and MEP, respectively. After IMT, the percentage of patients with MIP predicted for age in the T-IMT group increased to $21.1 \%$ and in the I-IMT group to $40 \%$. These results tie in with several studies showing a reduction in respiratory muscle strength and changes in lung function of patients on HD compared with the predicted values for age or healthy subjects $^{(22-24)}$. Other studies have evaluated the acute effects of $\mathrm{HD}$ on strength and lung function and showed that the weight gained between dialysis sessions has deleterious effects on respiratory function ${ }^{(25,26)}$.

In the present study, we performed IMT for six months during HD sessions, with three sets of 10 inspirations (30\% MIP) initially and then with three sets of 14 inspirations (50-60\% of MIP) at the end; our results were satisfactory for both groups. To obtain findings for the effects of resistance training on the respiratory muscles, beyond the Principle of Overload, the specificity (inspirations of high intensity and short duration) was taken into consideration ${ }^{(27)}$. In the literature, three published studies have evaluated the effects of IMT on patients receiving $\mathrm{HD}^{(10-12)}$ only one study was randomized and controlled (10 weeks of treatment, three sets of 15 inspirations, three times per week and during hemodialysis) and showed a significant increase in respiratory muscle strength and in aspects related to quality of life ${ }^{(12)}$. The improvement in respiratory strength in this study was probably owing to adaptations by the inspiratory muscles linked to the type of training (moderate load) that promotes hypertrophy (increase in muscle strength), increases the proportion of type I fiber, reduces type II fiber and increases 
Table 3- Generic and specific dimensions of the Kidney Disease Quality of Life-Short Form pre- and post-inspiratory muscle training.

\begin{tabular}{|c|c|c|c|c|c|c|}
\hline \multirow{2}{*}{ Dimensions ( $\mathrm{n}$ - of items) } & \multicolumn{2}{|c|}{$\begin{array}{l}\text { T-IMT } \\
(n=19)\end{array}$} & \multicolumn{2}{|c|}{$\begin{array}{l}\text { I-IMT } \\
\text { (n=15) }\end{array}$} & \multicolumn{2}{|c|}{$\begin{array}{l}\text { IH-IMT } \\
(n=16)\end{array}$} \\
\hline & Pre & Post & Pre & Post & Pre & Post \\
\hline Physical functioning (10) & $70.3(24.4)$ & $69.0(24.9)$ & $72.3(25.5)$ & $72.7(29.5)$ & $71.2(29.0)$ & $71.8(31.3)$ \\
\hline Physical role (4) & $75.8(38.3)$ & $85.5(34.7)$ & $57.0(46.5)$ & $83.3(32.3)^{*}$ & $78.0(41.3)$ & $70.6(42.6)$ \\
\hline Pain (2) & 74.4 (29.9) & $77.6(30.0)$ & $89.6(19.8)$ & $77.5(23.0)$ & $74.4(24.9)$ & $90.7(12.6)$ \\
\hline Health general (5) & $63.2(26.6)$ & $56.6(29.6)$ & $70.1(19.8)$ & $59.7(22.6)^{*}$ & $64.4(29.5)$ & $59.7(30.6)$ \\
\hline Emotional wellbeing (5) & $75.4(28.1)$ & $77.3(26.1)$ & $78.8(18.8)$ & $81.9(17.9)$ & $72.9(17.7)$ & $85.9(15.2)$ \\
\hline Emotional role (3) & $60.2(44.8)$ & $78.9(37.2)$ & $78.7(32.4)$ & $86.7(27.6)$ & $86.3(33.5)$ & $84.3(35.6)$ \\
\hline Social function (2) & $73.8(26.7)$ & $88.2(19.3)^{*}$ & $85.3(25.0)$ & $83.7(23.3)$ & $76.6(27.3)$ & $86.7(22.6)$ \\
\hline Energy/fatigue (4) & $66.3(23.8)$ & $68.7(27.7)$ & $69.7(21.7)$ & $68.8(21.2)$ & $64.4(22.6)$ & $72.6(19.0)$ \\
\hline List of sintoms/problems (12) & $87.1(13.2)$ & $90.8(10.4)$ & $84.8(14.4)$ & $87.4(11.3)$ & $87.5(13.7)$ & $87.6(13.0)$ \\
\hline Effects of kidney desease (8) & $65.0(25.5)$ & $64.6(27.4)$ & $71.9(18.3)$ & $71.7(16.8)$ & $78.1(14.4)$ & $74.3(19.3)$ \\
\hline Burden of kidney desease (4) & $41.8(30.3)$ & $42.4(32.2)$ & $45.8(28.1)$ & $52.5(27.6)$ & $50.0(34.3)$ & $55.1(27.7)$ \\
\hline Work (2) & 39.5 (39.4) & $36.8(36.8)$ & $40.0(43.1)$ & $50.0(46.3)$ & $35.3(38.6)$ & $44.1(46.4)$ \\
\hline Cognitive function (3) & $82.5(19.7)$ & $90.5(15.8)^{*}$ & $89.3(10.3)$ & $88.9(13.7)$ & $91.4(14.3)$ & $96.1(5.8)$ \\
\hline Social interaction (3) & $85.3(15.6)$ & $87.0(17.0)$ & $95.1(7.3)$ & $93.8(7.8)$ & $90.6(17.6)$ & $91.0(12.5)$ \\
\hline Sexual function (2) & $36.2(46.6)$ & $61.2(44.1)^{*}$ & $54.2(46.7)$ & $58.3(45.2)$ & $36.0(47.6)$ & $35.3(46.8)$ \\
\hline Sleep (4) & $74.6(23.9)$ & $73.8(26.4)$ & $77.0(14.7)$ & $80.1(18.3)$ & $74.1(22.7)$ & $77.6(28.2)$ \\
\hline Social support (2) & 89.5 (15.9) & $90.2(25.6)$ & $75.6(30.1)$ & $74.4(41.2)$ & $84.3(26.7)$ & $85.3(23.5)$ \\
\hline Dialysis staff (2) & $92.8(17.8)$ & $86.8(26.5)$ & $95.8(13.1)$ & 96.7 (12.9) & $87.5(17.1)$ & $93.4(16.6)$ \\
\hline Patient satisfaction (1) & $78.1(18.5)$ & $72.8(20.9)$ & $72.2(19.6)$ & $74.4(17.7)$ & $70.1(18.0)$ & $82.4(18.1)$ \\
\hline
\end{tabular}

Note: T-IMT = trainer - inspiratory muscle training; I-IMT = incentive - inspiratory muscle training. Values of $p$ obtained to $p<0.05$.

the cross-sectional area in the diaphragm ${ }^{(7,8)}$. These changes are reflected in an increase in inspiratory muscle strength as assessed by MIP.

With respect to the spirometrics variables, we found no significant difference after IMT, except to PEF in the T-IMT group (Table 3). Studies have shown that IMT does not influence spirometrics variables and perhaps the application of endurance training may have effects on lung function, since exercises are performed in high flows and tidal volumes ${ }^{(11,12)}$. The fluid overload between HD sessions and abnormal permeability of the pulmonary microcirculation have been cited as causes of acute pulmonary edema (noncardiogenic) and pleural effusion (28) that can reduce volumes and lung capacity over time. This respiratory complication changes the ventilation/perfusion relationship (with or without the presence of anemia), reduces gas exchange, tissue oxygenation, and, consequently, functional capacity (activities of daily living) and exercise tolerance ${ }^{(29-31)}$.

In the present study, significant improvements in the dimensions of the SF-KDQOL showed that IMT contributed to an increase in the scores of cognitive and social function. Scores for the dimensions of sexual function in the T-IMT group and physical function in the I-IMT group also increased after training (Table 3). Sexual function is particularly impaired in HD patients due to physical factors (intermittent uremia, continuous anemia, hormonal and gonadal dysfunction and spermatogenesis damage in the case of males), psychological factors (depression and anxiety reduce interest and ability in daily activities, including sex) and drug interactions (use of antihypertensive drugs, which leads to reduced libido and impotence in men) which interfere with sexual activity ${ }^{(14,32-33)}$. However, studies have demonstrated that these factors can be influenced by a training program (resistance exercises, aerobic training and flexibility) which can improve the health-related quality of life of HD patients. ${ }^{12,34,35)}$ The training program should not be neglected in health care because it has a positive effect on mental health and well-being, reducing depression and anxiety and improving cognitive functioning ${ }^{(36)}$.

Some aspects about the relevance of this study should be highlighted. First, there has been no mention in the literature of IMT in HD patients for a long time (six months) ${ }^{(10,12)}$. Secondly, the increase in inspiratory muscle strength in these patients may make them less susceptible to respiratory complications caused by the kidney disease itself (general muscle mass loss, respiratory included) and the weight gained between dialysis sessions that commonly increases the number of admissions 
of these patients into hospital (acute pulmonary edema and pleural effusion). It has been possible to show that the least expensive device (incentive spirometer) for performing IMT was also the one that offered the most increments of muscle strength after training.

Although IMT showed significant increases in inspiratory muscle strength, our study had some limitations: the small number of patients allocated to each group (reduced adhesion of patients), the large number of co-morbidities that were inclusion criteria for the study and the loss of patients during the study due to several factors (transfer unit, death, surgery and dropouts). Other aspects should also be considered, such as the lack of a control group in all stages of intervention and the lack of females included in the study. In addition, the device used to train the T-IMT group is costly and its use is impractical in clinical care. In the present study, it was not possible to evaluate specific biochemical parameters (CPK and albumin) to check muscle breakdown and the availability of fatty acids as a source of substrate for exercising muscle evaluated by albumin. Further studies are suggested with IMT (interventions longer than six months) and associated resistive or aerobic training to verify the benefits of exercise in reducing respiratory complications, to enhance functional capacity and quality of life in HD patients.

\section{CONCLUSION}

In conclusion, the study showed that respiratory muscle strength and pulmonary function are reduced in male patients receiving hemodialysis. However, it was proved that at least three months of IMT, with the use of different types of apparatus, is able to improve inspiratory muscle strength in both groups as measured by MIP. Also, I-IMT promoted increments significantly larger in MIP. Furthermore, it was demonstrated that T-IMT significantly improved health-related quality of life aspects (sexual, social and cognitive function), while I-IMT ameliorated the physical function of male HD patients.

\section{AUTHOR DETAILS}

${ }^{2}$ Health of Science Post Graduate Program, Universidade Federal de Goiás (UFG), Goiânia, GO, Brazil

\section{REFERENCES}

1. Kopple JD, Storer T, Casburi R. Impaired exercise capacity and exercise training in maintenance hemodialysis patients. Journal of renal nutrition. 2005; 15 (1):44-48.

2. Painter P. Physical functioning in end-stage renal disease patients: update 2005. hemodialysis international. International Symposium on Home Hemodialysis. 2005; 9 (3):218-235.

3. Caimi G, Caimi G, Carollo C, Lo Presti R. Pathophysiological and clinical aspects of malnutrition in chronic renal failure. Nutrition research reviews. 2005; 18 (1):89-97.

4. Kaysen GA, Greene T, Larive B, et al. The effect of frequent hemodialysis on nutrition and body composition: Frequent hemodialysis Network Trial. Kidney international. 2012; 82 (1):90-99.
5. Smogorzewski M, Piskorska G, Borum PR, Massry SG. Chronic renal failure, parathyroid hormone and fatty acids oxidation in skeletal muscle. Kidney Int. 1988; 33 (2):555-560.

6. Gordon PL, Sakkas GK, Doyle JW, Shubert T, Johansen KL. Relationship between vitamin $D$ and muscle size and strength in patients on hemodialysis. Journal of Renal Nutrition. 2007; 17 (6):397-407.

7. Kouidi E, Albani M, Natsis K, et al. The effects of exercise training on muscle atrophy in haemodialysis patients. Nephrology Dialysis Transplantation. 1998; 13 (3):685-699.

8. Workeneh BT, Rondon-Berrios H, Zhang L, et al. Development of a diagnostic method for detecting increased muscle protein degradation in patients with catabolic conditions. J Am Soc Nephrol. 2006; 17 (11):3233-3239.

9. Lewis MI, Fournier M, Wang $\mathrm{H}$, et al. Metabolic and morphometric profile of muscle fibers in chronic hemodialysis patients. J Appl Physiol. 2012; $112(1): 72-78$.

10. Weiner P, Ganem R, Zamir D, Zonder H. Specific inspiratory muscle training in chronic hemodialysis. Harefuah. 1996;130(2):73-76, 144.

11. Silva VGd, Amaral C, Monteiro MB, Nascimento DMd, Boschetti JR. Efeitos do treinamento muscular inspiratório nos pacientes em hemodiálise; Effects of inspiratory muscle training in hemodialysis patients. J. bras. nefrol. 2011; 33(1):62-68.

12. Pellizzaro CO, Thome FS, Veronese FV. Effect of peripheral and respiratory muscle training on the functional capacity of hemodialysis patients. Renal failure. 2013; 35 (2):189-197.

13. Daugirdas JT. Second Generation Logatithmic Estimates of Single-Pool Variable Volume Kt/V: An Analyses of Error. Journal American of Society of Nephrology. 1993;14: 1205-1213.

14. Duarte PS, Ciconelli RM, Sesso R. Cultural adaptation and validation of the "Kidney Disease and Quality of Life--Short Form (KDQOL-SF 1.3)" in Brazil. Brazilian Journal of Medicine and Biology Research. 2005; 38(2):261-270.

15. Black LF, Hyatt RE. Maximal respiratory pressures: normal values and relationship to age and sex. American Review of Respiratory Disease. 1969; 99 (5):696-702.

16. Neder JA, Andreoni S, Lerario M, Nery L. Reference values for lung function tests: II. Maximal respiratory pressures and voluntary ventilation. Brazilian Journal of Medical and Biological Research. 1999; 32 (6):719-727.

17. Neder JA, Andreoni S, Lerario MC, Nery LE. Reference values for lung function tests. II. Maximal respiratory pressures and voluntary ventilation. Brazilian Journal of Medicine and Biology Research. 1999; 32 (6):719-727.

18. Miller MR, Hankinson J, Brusasco V, et al. Standardisation of spirometry. European Respiratory Journal. 2005; 26 (2):319-338.

19. Neder JA, Andreoni S, Castelo-Filho A, Nery LE. Reference values for lung function tests: I. Static volumes. Brazilian Journal of Medical and Biological Research. 1999; 32:703-717.

20. Chuter TA, Weissman C, Mathews DM, Starker PM. Diaphragmatic breathing maneuvers and movement of the diaphragm after cholecystectomy. Chest. 1990; 97 (5):1110-1114.

21. Kisner C, Colby LA. Therapeutic exercise: foundations and techniques. Philadelphia: F. A Davis Company; 2002.

22. Cury JL, Brunetto AF, Aydos RD. Efeitos negativos da insuficiência renal crônica sobre a função pulmonar e a capacidade funcional. Revista Brasileira de Fisioterapia. 2010;14(2):91-98.

23. Bakirci T, Sasak G, Ozturk S, Akcay S, Sezer S, Haberal M. Pleural effusion in long-term hemodialysis patients. Paper presented at: Transplantation proceedings 2007.

24. Bavbek N, Akay H, Uz B, et al. The effects of L-carnitine therapy on respiratory function tests in chronic hemodialysis patients. Renal failure. 2010; 32 (2):157-161. 
25. Kovačević P, Matavulj A, Veljković S, Rajkovača Z, Ponorac N, Huskić J. Ventilator function improvement in patients undergoing regular hemodialysis: relation to sex differences. Bosnian Journal of Basic Medical Sciences. 2006; 6 (1):29-32.

26. Karacan O, Tutal E, Uyar M, Eyuboglu FO, Sezer S, Ozdemir FN. Pulmonary function in uremic patients on long-term hemodialysis. Renal failure. 2004; 26 (3):273-278.

27. McConnell A. In: McConnell A, ed. Respiratory Muscle Training. Oxford: Churchill Livingstone; 2013:135-147.

28. Bush A, Gabriel R. Pulmonary function in chronic renal failure: effects of dialysis and transplantation. Thorax. 1991; 46 (6):424-428.

29. Bush A, Gabriel R. The lungs in uraemia: a review. Journal of the Royal Society of Medicine. 1985; 78 (10):849.

30. Gehm L, Propp DA. Pulmonary edema in the renal failure patient. The American journal of emergency medicine. 1989; 7 (3):336-339.

31. Rashid-Farokhi F, Pourdowlat G, Nikoonia MR, et al. Uremic pleuritis in chronic hemodialysis patients. hemodialysis international. International Symposium on Home Hemodialysis. 2013; 17 (1):94-100.
32. Levy NB. The sexual rehabilitation of the hemodialysis patient. Sexuality and Disability. 1979; 2 (1):60-65.

33. Korevaar JC, Merkus MP, Jansen MA, Dekker FW, Boeschoten EW, Krediet RT. Validation of the KDQOL-SF: a dialysis-targeted health measure. Quality of life research: an international journal of quality of life aspects of treatment, care and rehabilitation. 2002;11(5):437-447.

34. De Moura Reboredo M, Henrique DMN, De Souza Faria R, Chaoubah A, Bastos MG, De Paula RB. Exercise training during hemodialysis reduces blood pressure and increases physical functioning and quality of life. Artif Organs. 2010; 34 (7):586-593.

35. Painter P, Moore G, Carlson L, et al. Effects of exercise training plus normalization of hematocrit on exercise capacity and health-related quality of life. Am J Kidney Diseas. 2002; 39 (2):257-265.

36. Callaghan P. Exercise: a neglected intervention in mental health care? Journal of psychiatric and mental health nursing. 2004;11(4):476483. 